\title{
Natural ACTH Relieves Acute Inflammation of Gout by Changing the Function of Macrophages
}

\section{Rui Xu}

Huashan Hospital Fudan University

\section{Li Zhao}

Huashan Hospital Fudan University

\section{Jiyu Liu}

Huashan Hospital Fudan University

\section{Lin Cao}

Huashan Hospital Fudan University

\section{Tianyi Zhao}

Huashan Hospital Fudan University

\section{Yiyun Yu}

Huashan Hospital Fudan University

\section{Dandan Xuan}

Huashan Hospital Fudan University

\section{Weiguo Wan}

Huashan Hospital Fudan University

\section{Hejian Zou}

Huashan Hospital Fudan University

\section{YuXue ( $\nabla$ yxue@unirheuma.org )}

Fudan University https://orcid.org/0000-0002-4575-9243

\section{Research article}

Keywords: natural ACTH, macrophages, acute gout, corticosteroids

Posted Date: August 20th, 2021

DOI: https://doi.org/10.21203/rs.3.rs-797094/v1

License: (9) This work is licensed under a Creative Commons Attribution 4.0 International License. Read Full License 


\section{Abstract}

Objective: Gout is a common arthritis caused by deposition of monosodium urate crystals. Macrophage is crucial in the process of monosodium urate (MSU) -induced inflammation. Although it has been reported that adrenocorticotropic hormone (ACTH) in nature can be used to cure urarthritis, the mechanism concerning macrophage is still not clear. This study aims to explore how natural ACTH can alleviate urarthritis through functional changes in macrophage.

Methods: We analysed the variations in VAS pain scores of five patients, knowing the time of action, and detecting the level of cortisol and ACTH in patients 24 hours after the application of ACTH. The effect of natural ACTH on joint inflammation and the level of cortisol in blood in mouse model was evaluated by studies in vivo. In vitro studies we evaluated the effect of natural ACTH on macrophage and revealed different functions of ACTH and dexamethasone on macrophage in the transcriptional level.

Results: In patients with acute gout, natural ACTH can quickly alleviate pain and has no effect on the level of cortisol and ACTH. Natural ACTH is able to ease the swelling and inflammatory cell infiltration caused by arthritis, without changing the level of cortisol. Besides, natural ACTH in vitro can alleviate acute gouty inflammation by regulating phagocytosis and polarization of macrophage, which also exert different effects on the transcription of some related genes.

Conclusion: Natural ACTH is able to alleviate acute gouty inflammation by regulating macrophage, and this effect differs from that of dexamethasone in the transcriptional level.

\section{Introduction}

Gout is a kind of crystal related arthropathies, caused by deposition of monosodium urate (MSU) in joints. It is directly related to hyperuricemia caused by purine metabolic disorders and/or decreased excretion of uric acid, making it a kind of metabolic rheumatism(1). Apart from joint damage, patients with gout may also have kidney disease and other metabolic syndromes, such as hyperlipidemia, hypertension, diabetes and coronary heart disease, etc(2). It strongly recommends the colchicine, nonsteroidal anti-inflammatory drugs (NSAIDs) or corticosteroids (oral, intra-joint or muscle injection) being seen as the first-line drugs for the treatment of acute gout by 2020 American College of Rheumatology Guildlines for the Management of Gout(3). However, patients with gout may have other complications such as hypertension, diabetes, chronic kidney disease and hormone intolerance, etc. Therefore, some first-line drugs may not be effective and even cannot be used for treatment, which poses a great challenge to the treatment of gout.

Adrenocorticotropic hormone, ACTH, is a kind of polypeptide hormone secreted by pituitary gland of vertebrates. It can promote the tissue hyperplasia of adrenal cortex and the generation and secretion of cortical hormone. Natural ACTH, whose generation and secretion are directly regulated by corticotropinreleasing factors (CRF) secreted from hypothalamus(4), is an important hormone in terms of maintaining the normal morphology and function of the adrenal gland. At present, natural ACTH is commonly used to 
treat collagenosis like rheumatoid arthritis(5-6) and systemic lupus erythematosus(7-8), while it does not serve as a first-line drug for the treatment of acute gout. In fact, ACTH was first used to treat acute gout more than half a century ago(9). Since then, some studies showed that ACTH had equal effects with NSAIDs and steroids, and had a safety profile(10-12). Besides, gout patients who were treated with ACTH had a stable level of blood pressure and serum potassium(13), without showing apparent adverse reactions. A study showed that ACTH was able to be used to treat gout patients who had various complications or who could not bear standard treatment, and the effective rate could be as high as 77.9$100 \%(14)$. Previous studies believed that inflammatory factors like IL-I 3 , secreted when there is acute gouty arthritis, stimulate anterior pituitary gland to generate natural ACTH. The ATCH can bind to the receptor, MC-2R, on adrenal and therefore induce the secretion of corticosteroid, finally leading to the downregulation of inflammatory reaction(14-15). Studies in recent years have extended our knowledge involving the mechanism of acute gout. It is said that ACTH can also bind to receptor MC-3R on macrophages to stimulate the transduction of melanocortin and reduce the release of chemokines which are responsible for the accumulation of neutrophil(14), thereby alleviating inflammation. It also has less adverse effect compared to cordicosteroids. However, it is still unclear how natural ACTH affects the function of macrophage, and it is crucial to further study the mechanism.

The aim of this study is to explore the mechanism by which natural ACTH alleviates inflammation caused by acute gout through macrophage. We conducted studies both in vivo and in vitro, finding that natural ACTH can alleviate the inflammation existing in the joints of mice with acute gout, and is able to affect the polarization and phagocytosis of macrophage to some extent, which functions in different ways in contrast to typical cordicosteroids like dexamethasone.

\section{Materials And Methods}

\subsection{Patients}

Five gout patients included in the study were all recorded in the Department of Rheumatology in Huashan Hospital affiliated to Fudan University. We have had an adequate intercommunication with patients and treated them with natural ACTH after reaching an agreement.

\subsection{Mice}

Six-week-old C57BL/6 mice were bought from Shangahi JieSiJie Laboratory Animal Limited Corporation and were fed under sterile conditions. Experiments involving animals were conducted according to protocols approved by the Animal Committee of Fudan University.

\subsection{Histopathology}

The mice paw was cut into $5 \mu \mathrm{m}$ slices and embedded with paraffin so as to do hematoxylin-eosin (HE) staining.

\subsection{ELISA}


ELISA kit from eBioscience was used to detect the concentration of serum cortisol and factors like IL-1 $\beta$, IL-6, TNF- $a$, TGF- $\beta$ in the supernatant of cells.

\subsection{Natural ACTH}

Natural ACTH (25U per ampoute) was purchased from Shanghai NO.1 Biochemical \& Pharmaceutical Limited Corporation. Triple distillation $\mathrm{H}_{2} \mathrm{O}$ was used to dissolve it into different concentrations according to the design of experiment.

\subsection{Cell culture and pre-treatment}

THP1 cells were bought from National Collection of Authenticated Cell Cultrues and kept in the RPMI 1640 culture medium containing 10\% FBS in humid conditions with $5 \% \mathrm{CO} 2$. The cells were subcultured every 48 to 72 hours. When there had enough cells, the cells were transferred to cell culture plates. PMA was added at a ratio of 1:10000 for 48 hours so as to induce cultured cells into macrophages. Culture medium was changed before experiment and was disposed by different methods according to specific experiments.

\section{7. $\mathrm{qPCR}$}

TRIzol was used to extract total RNA and ReverTraAce qPCR RT kit (Toyobo) was used for reverse transcription. qPCR was conducted on CFX96 Touch Real-Time PCR detection system (Bio-Rad) with the application of SYBR Green Kit (Roche). Primer sequences:

5'-CCAAATTGCTGCATGAACCAG-3'(forward) 5'-TGCTTCCGAGGAGTGTCTTTC-3'(reverse) (for XDH gene);

5'-TGCTGCCCTTTGACAACCTG-3'(forward) 5'-TGCTCCCGAAGTAAGAGGGT-3'(reverse)(forMPOgene); 5'TTGTTTGGTTAGGGCTGAATGT-3'(forward) 5'-GCCAATGTTGACCCAAGGATTTT-3'(reverse) (forNOX1gene); 5'-ACCGTGGAGGAGGCAATTAGA-3'(forward) 5'-TGGTTGCATTAACAGCTATCCC3 '(reverse) (for NOX3 gene);

5'-AGAAGATTCGGAAACAACAGCA-3'(forward) 5'-GCTGGATCATTAGTTCTTGAGCC-3'(reverse) (for NR1H2 gene);

5'-TTCTTTGCATTAGCCTCATCCTT-3'(forward) 5'-CGTGCTGATTCCTTTTGGGC-3'(reverse) (for IL-37 gene);

5'-GATCTTCGCTGCGATCAACAG-3'(forward) 5'-CGTGCATTATCTGAACCCCAC-3'(reverse) (for NLRP3 gene);

5'-GACTTTAAGGGTTACCTGGGTTG-3'(forward) 5'-TCACATGCGCCTTGATGTCTG-3'(reverse) (for IL-10 gene);

5'-CTCTGGCGTAGAGCTATCACT-3'(forward) 5'-AGGCTGGGTTGGTGAAAACA-3'(reverse) (for MerTK gene); 5'-CCTCCTGTCTAGTCGGTTTGG-3'(forward) 5'-TCGAGCACCCTGTACCATTGA-3'(reverse) (for FcyRIIIA gene); 
5'-CATCTCCTTGCGATTCTGTTTTG-3'(forward) 5'-CCATTCCGAGTGCTCCTGA-3'(reverse) (for PTX3 gene);

5'-GACTGTCCTCGTGTGGTTTTG-3'(forward) 5'-GGCTGCCCAGCATATCAGAT-3'(reverse) (for MC2R gene);

5'-GCCAACACTGCCTAATGGCT-3'(forward) 5'-AACCTCGGGCTTGATGAAGAC-3'(reverse) (for MC3R gene);

5'-TCTGAGTCTGTATGGAGTGACAT-3'(forward) 5'-CCAAGTCGTTCACATCTAGTTCA-3'(reverse) (for PGC1a gene);

5'-TGGACGCAGGTTCTCCAAAC-3'(forward) 5'-CCGGCTCGCAGTAGGTAAC-3'(reverse) (for SMAD3 gene);

5'-GGTAGCTGAGTTTGACTTCCG-3'(forward) 5'-GACAGCATCCCTGTTGACCTT-3'(reverse) (for GAS6 gene).

\subsection{Flow cytometry}

Single cell suspension was stained by anti-Arg-1 (17-3697-82; eBioscience) and anti-iNOS (12-5920-82; eBioscience) antibody. The antibodies were used at a ratio of 1:100, incubating for 40 minutes without light, with the temperature kept at $4{ }^{\circ} \mathrm{C}$. FITC-Latexbeads (500290; caymanchem) were added immediately when the experiment began. Canton $\mathrm{C} 6$ flow cytometer was used to conducted the experiment and collected data were analyzed by Flowjo.

\subsection{Intracellular ROS determination}

Reactive Oxygen Species Assay Kit (DCFH-DA, Beyotime, Jiangsu, China) was used to detect the level of intracellular ROS. After stimulation, the culture medium was removed, and DCFH-DA was added to serumfree medium at a ratio of 1:1000 for cell incubation, which lasted for 20 minutes with the temperature kept at $37^{\circ} \mathrm{C}$. Then after being washed by serum-free medium for 3 times, cells were observed and detected by fluorescence microscope and flow cytometer, respectively.

\subsection{Determination of mitochondrial function}

Variations involving the opening of mitochondrial permeability transition pore (mPTP) were detected by Mitochondrial Permeability Transition Pore Assay Kit (Beyotime, Jiangsu, China) in order to determine the functions of mitochondrion. After stimulation, culture medium was removed and PBS was used to wash the cells for 1 to 2 times. Proper amount of Calcein AM was added to incubate the cells for $30-45$ minutes at $37^{\circ} \mathrm{C}$ without light. Then fresh medium was applied for another 30 -minute incubation at 37 ${ }^{\circ} \mathrm{C}$ without light. Finally, the medium was removed and cells were washed 2-3 times with PBS before obeservation under a fluorescence microscope and detection using a flow cytometer.

\section{Statistical Analysis}

Data collected were analyzed by unpaired student's $t$ test in Graphpad Prism. It is considered significantly different if the $P$ value is less than 0.05 . 


\section{Results}

4.1. Natural ACTH can quickly alleviate inflammation caused by acute gout, without affecting the level of cortisol and ACTH in patients.

VAS pain scale was used to evaluate the pain suffered by 5 male patients who were treated with natural ACTH. All patients sufferred less to some extent 6-12 hours on average after the application of the drug. The level of cortisol and ACTH in blood lied in normal range 24 hours after the drug application. An additional file shows this in more detail (see Additional file 1).

4.2. The swelling and inflammation shown in the paw of mice model can be alleviated by natural ACTH, without evident effect on the level of cortisol.

MSU crystal was injected through the mice paw to make models of acute gouty arthritis for detection. The right paw showed significant swelling after solely injecting MSU crystal when compared to the control group and the other paw of treated mice. Subcutaneous injection of ACTH at a concentration of $0.25 \mathrm{U} / \mathrm{ml}$ per mouse had no effect on the swelling of paw while $2.5 \mathrm{U} / \mathrm{ml}$ per mouse was able to alleviate it (Fig. 1A). Severe inflammation was observed in the MSU injected group by HE staining, while mice treated with natural ACTH had less inflammation. (Fig. 1B). No significance was shown within groups when detecting the concentration of cortisol in blood by ELISA (Fig. 1C).

\subsection{Natural ACTH can alleviate inflammation caused by actue gout by changing the function of macrophage.}

\subsubsection{Natural ACTH weakens the phagocytosis of macrophage in terms of MSU and Beads. We added} natural ACTH with different concentrations into THP1 cells, using dexamethasone as the control drug, to evaluate the changes of phagocytosis of THP1 cells. Both natural ACTH and dexamethasone could inhibit the ability of phagocytosis when compared with the experimental group where MSU was added solely. When the concentration of natural ACTH was higher, the effect of inhibition was more severe (Fig. $2 \mathrm{~A})$. Both natural ACTH and dexamethasone could inhibit the ability of macrophage to engulf FITClatexbeads in contrast with the control group while higher concentration of natural ACTH had stronger effect of inhibition (Fig. 2B).

\subsubsection{Natural ACTH can promote the macrophage $M 2$ polarization and downregulate the level of pro-inflammatory factors.}

Later, we detected the polarization of macrophage $\mathrm{M} 2$ and the level of related inflammatory factors. THP1 cells can be stimulated by MSU crystal to release inflammatory factors like IL-1 $\beta$ (Fig. 3A), IL-6(Fig. 3B) and TNF-a(Fig. 3C). The application of natural ACTH and dexamethasone could significantly lower the level of these factors. When natural ACTH existing, the level of TGF- $\beta$ did not change. Only when dexamethasone was applied, the level of TGF- $\beta$ was decreased (Fig. 3D). When using flow cytometry to 
detect and analyze the polarization markers like iNOS/Arg-1, we found that THP1 cells could be stimulated by MSU crystal and display significant M1-type polarization. Low concentration of natural ACTH could inhibit the trend and promote macrophage to polarize to M2 type, while high concentration of natural ACTH led to no significant change (Fig. 3B).

\subsubsection{Natural ACTH can downregulate the ROS level of gouty macrophages and protect the function of mitochondrion, which can be related to the inhibition of XDH generation.}

We studied microchondrion-related function of THP1 cells. As shown in Fig. 4A, MSU crystal could significantly increase the generation of ROS, while both natural ACTH and dexamethasone could inhibit the process. We further explored whether natural ACTH can protect the mitochondrion by detecting the opening of MPTP, which could be promoted by MSU crystal as shown in Fig. 4B. The function could be reversed by natural ACTH and dexamethasone. Besides, the protective effect of natural ACTH was stronger than that of dexamethasone, and had a positive correlation with its concentration. When detecting the genes that are related to the generation of ROS, the group treated with natural ACTH displayed lower mRNA expression of XDH, while the dexamethasone group displayed lower mRNA expression of NOX3 (Fig. 4C).

\subsection{Natural ACTH has a different anti-inflammatory mechanism from dexamethasone on the transcription of Inflammatory-related genes.}

PCR array was used to analyze transcriptional differences existing between natural ACTH and dexamethasone when concerning anti-inflammation (Fig. 5A). Figure 5B to Fig. 5J showed differences between natural ACTH and dexamethasone in metabolism (Fig. 5B), inflammation (Fig. 5C), phagocytosis (Fig. 5D), melanocortin receptors (Fig. 5E) and some other related genes (Fig. 5F-Fig. $5 \mathrm{~J}$ ). The expression of most genes was not affected by the addition of natural ACTH, though there were some up-regulated genes like NR3C1, NR1H2, IL-37, FcyRIIIA and GAS6 together with some downregulated genes like NLRP3, MC2R and MC3R. Dexamethasone showed significantly differences in the expression of ANGPTL4, PDP1, PDHA1, VDR, IL1RN, IL-10, Mertk, PTX3, DDIT4 and SMAD3 compared with natural ACTH.

\section{Discussion}

It can be traced to half a century ago when natural ACTH was applied to treat acute gout. Lots of studies have shown, natural ACTH can improve the function of kidney and have lipid-lowering effect(15) when compared to traditional medicine like NSAIDs and corticosteroids. Besides, ACTH can be used to treat hypoadrenia, glucocorticoid resistance and even those who have contraindications. Currently, natural ACTH is seldom used to treat acute gout because the available formulation of ACTH in most countries bears a high cost. We conducted experiments concerning the treatment of acute gout using natural ACTH, exploring its application in the treatment of acute gouty arthritis in mice and its function of regulation in 
mediating THP1 cells, which is stimulated by MSU. In the animal experiment, we found highconcentration of natural ACTH, when subcutaneously injected into the mice, could effectively alleviate the paw swelling and inflammation caused by MSU crystal stimulation. Although low-concentration of natural ACTH may not ameliorate the swelling, it could still prevent the accumulation of inflammatory cells. In the meantime, the level of cortisol in mice blood did not show significant changes, indicating that the application of natural ACTH will not affect the level of cortisol in mice.

When acute gout attacks patients, macrophages within articular cavity and those transformed from monocyte existing in blood will engulf MSU, secreting pro-inflammatory factors to induce inflammation(16), and thus playing an important role in the progression and development of inflammation. When focusing on this aspect, we added natural ACTH and dexamethasone into THP1 cells which had been stimulated by MSU, finding that both of them were able to inhibit the THP1 cells when considering its phagocytic function on MSU crystal. High concentration of natural ACTH could strongly inhibit the cells, the effect of which could be similar to dexamethasone. In order to verify that the inhibitory function on THP1 cells does not specifically aimed at MSU, we used FITC-latexbeads to detect the phagocytic ability of THP1 cells. The latexbeads with FITC could be engulfed by macrophage, as detected by fluorescence microscope or flow cytometry. The results are similar to that with MSU stimulation, which confirms that natural ACTH is able to inhibit the phagocytosis of THP1 cells and further prevent the MSU-stimulated secretion of inflammatory factors.

Apart from phagocytosis, macrophage will develop into pro-inflammatory M1-type macrophage during the early period of inflammation(17). MSU crystal can stimulate the cells to generate IL-1 $\beta$, IL-6, TNF-a and induce inflammatory infiltration, driving the persistence of gouty inflammation(18). Our previous results showed that natural ACTH could alleviate the inflammation in the mice paw and weaken the phagocytosis of macrophage, which may be caused by the polarization of M2-type macrophage promoted by natural ACTH as we implied. Unsurprisingly, natural ACTH inhibited the MSU-stimulated THP1 cells to secret M1-type inflammatory factors, the effect of which was equal to that of dexamethasone. In the meantime, when natural ACTH was added, Arg-1 in THP1 was expressed abundantly, suggesting that natural ACTH can inhibit inflammation by promoting M2-type polarization of THP1.

The function of immune cells depends on their metabolic activity to a large extent. Therefore, the cells need to develop metabolic adaptation so as to support their various immunological functions. Macrophage, as a crucial member participating in innate immunity, is strictly regulated by metabolic pathway and metabolites(19). We cannot ignore the role of mitochondrion, which is considered as the center for cell metabolism, in regulating immune cells. In recent years, more and more regulatory mechanisms have been gradually revealed. For example, mitochondrial reactive oxygen species (mtROS), generated alongside the process of electron transfer chain, may trigger innate immune signals and damage the cells, the extent of which depends on the volume of mtROS and when does it occur(20). Studies showed that inflammation may cause an increase of ROS in the macrophage mitochondrion and inhibit the function of oxidative phosphorylation(21-22), indicating the impaired function of 
mitochondrion. We implied that natural ACTH can protect the mitochondrion of THP1 cells to some extent, and carried out studies in this aspect. THP1 cells could generate much more ROS in the mitochondrion when stimulated by MSU, while natural ACTH and dexamethasone ccould inhibit the process with similar inhibitory effect. MPTP is a group of protein complex existing between the inner and outer membrane of mitochondria. It is a type of non-specific channel, playing a significant role in the survival and apoptosis of cells, and involved in various fields, such as ischemia/reperfusion, cancer, aging and neural degeneration(23). mPTP allows ions whose relative molecular mass is relatively low to permeate freely under physiological conditions. It can maintain the mitochondrial membrane potential and the balance of ions within and outside the cells by driving ATP synthase through oxidative phosphorylation. However, apoptotic signals will stimulate the mPTP to open thoroughly, making soluble matter permeate nonselectively, which then leads to the imbalance of ions and membrane potential depolarization, causing apoptosis or necrosis(24-25). mPTP can be used to detect the impairment of mitochondrional function, and thus we measured the protective function of natural ATCH on MPTP. Results showed that a great amount of MPTP existing in THP1 cells opened when being stimulated by MSU, indicating the function of mitochondrion was damaged. To the contrary, natural ACTH and dexamethasone reversed the phenomenon, and the function of natural ACTH was superior, suggesting natural ACTH is protective to mitochondrion in THP1 cells and maintains the functionality of MPTP. Meanwhile, we selected several genes, such as XDH, MPO, NOX1 and NOX3, all of which are associated with the generation of ROS to conduct qPCR test, and focused on XDH. Natural ACTH can inhibit the expression of XDH in MSU-stimulated THP1 cells, and XDH is able to turn products of purine metabolism into uric acid(26). Thus, we imply that natural ACTH can inhibit the generation of uric acid and protect the function of mitochondrion. However, more studies are needed to confirm the proposal.

When treating acute gout, patients who cannot react to colchicine or NSAIDs, or those who suffer from renal insufficiency, will always consider glucocorticoid as treatment. Although glucocorticoid are effective, it cannot be ignored that side-effects such as central obesity, infection, calcium loss, osteoporosis, diabetes, and stomach ulcers, etc.(27), will also bring inconvenience to patients. It is a big concern on how to largely avoid side-effect while receiving treatment. Here, a series of experiments found that natural ACTH was equal to dexamethasone when concerning their effect on treating acute gout. We still doubt whether there is difference existing between them when concerning the effect of treatment, and whether natural ACTH is a better choice for treating acute gout, which is also a question worth investigating. In addition to the results of the PCR array, we selectively chose some intriguing genes about metabolism, inflammation, phagocytosis and some others that may be involved during the process of exerting the anti-inflammatory effect. In metabolism-related genes, ANGPTL4 can mediate the inactivation of lipase in cells, causing hypetriglyceridemia and playing a role in lipid disorders, obesity and coronary heart disease(28). We here found that dexamethasone could significantly upregulate the transcription level of ANGPTL4, while ACTH had no evident effect. PDP1, pyruvate dehydrogenase phosphatase catalytic subunit 1 , can mediate the dephosphorylation and activation of pyruvate dehydrogenase, and therefore promote the tricarboxylic acid. PDHA1, pyruvate dehydrogenase a subunit, represents the key step for entering tricarboxylic(29). The intervention of Dexamethasone here 
significantly down-regulated the expression of PDP1 and PDHA1, suggesting that the application of glucocorticoid disturbed the normal procedure of saccharometabolism, while natural ACTH had no such effect. NR3C1 is a receptor of glucocorticoid within cells. In the presence of natural ACTH, its expression was up-regulated, which may because it has permissive effect on glucocorticoid. However, it was downregulated after dexamethasone intervention, which was attributed to the negative feedback. VDR is a nuclear receptor of calcitriol, and was detected to be down-regulated by dexamethasone intervention, indicating that hormones may affect the synthesis and catabolism of bones. In inflammatory-related genes, IL1RN is an antagonist of IL1 that can inhibit inflammation, and was found to be down-regulated by dexamethasone in our study. $\mathrm{NR} 1 \mathrm{H} 2$ plays an anti-inflammatory role during the hepatic acute phase response by acting as a corepressor and inhibits the hepatic acute phase response by preventing dissociation of the N-Cor corepressor complex(30); IL-37 is a suppressor of innate inflammatory and immune responses involved in curbing excessive inflammation(31), natural ACTH up-regulated their expression while dexamethasone did not, suggesting that two drugs may have different effects in antiinflammatory. As indispensable parts of the inflammatory response, the role of NLRP3 and IL-10 has long been known. Natural ACTH could down-regulated the expressiong of NLRP3 while had no such effect on IL-10, prompt that natural ACTH does not rely on IL-10 to exert its anti-inflammatory effect. In phagocytosis-related genes, MERTK is a kind of protein on the surface of macrophage which is able to activate macrophage and promote it to engulf apoptotic cells(32); PTX3 plays a role in the regulation of innate resistance to pathogens and inflammatory reactions(33); FcyRIIIA is a receptor for the Fc region of IgG which Binds complexed or aggregated IgG and also monomeric IgG. It mediates antibody-dependent cellular cytotoxicity (ADCC) and other antibody-dependent responses such as phagocytosis(34).

Dexamethasone up-regulated all of them while natural ACTH only up-regulated the expression of FcyRIIIA, suggests that they affect cell phagocytosis in different ways. MC2R and MC3R, as classic receptors of ACTH on macrophagesm, had been confirmed by many studies to be a key factor for ACTH to exert its anti-inflammatory effect through a steroid-independent pathway(14-15). We tested the expression of them after the stimulation of natural ACTH and dexamethasone, found that both of them were downregulated by natural ACTH, we speculate that it may be a result of some kind of negative feedback regulation which need to be further examined. In addition, we also investigatived some genes that may be involved in hormone and its receptor pathways. BCL6 is a crucial transcription factor, essential for the formation of lymphoid germinal center and maturity of antibody affinity(35), it is also a kind of protooncogene. It needs further confirmation in vivo whether the expression of BCL6 significantly downregulated by dexamethasone can be explained at the cellular level. DDIT4, a type of DNA damage induced transcription protein, is able to inhibit Rapamycin complex 1 and may affect cell proliferation, metabolism, autophagy and apoptosis through mTOR pathway(36). Hypoxia can significantly raise the level of DDIT, which is able to hinder the abnormal expression of downstream apoptotic proteins, and therefore to prevent apoptosis(36). Here, application of dexamethasone significantly induced its expression while natural ACTH did not show such effect. PGC-1a is a transcriptional coactivator for steroid receptors and nuclear receptors which plays an essential role in metabolic reprogramming in response to dietary availability through coordination of the expression of a wide array of genes involved in glucose and fatty acid metabolism(37);SMAD3 is an intracellular signal transducer and transcriptional 
modulator activated by TGF- $\beta$ and activin type 1 receptor kinases, also acts as a regulator of chondrogenesis and osteogenesis and inhibits early healing of bone fractures(38), Nactural ACTH showed no significant change while dexamethasone up and down-regulated their expression respectively, suggested that the use of dexamethasone may affect the body's normal function of physiological metabolism and healing. GAS6 is a ligand for tyrosine-protein kinase receptors AXL, TYRO3 and MER whose signaling is implicated in cell growth and survival, cell adhesion and cell migration(39). The expression of GAS6 upregulated by natural ACTH might suggest that it has the ability of protecting cells'normal function. After testing and analyzing the genes above, we believed that natural ACTH had a similar therapeutic effect as dexamethasone, while also protects cells and does not have a major impact on the body's metabolism. It seemed that natural ACTH were doing well in treating acute gout inflammation while avoiding the side effects of drugs.

To summarize, we explored the function of natural ACTH in treating acute gout, which includes alleviating the inflammation and regulating macrophage. At the same time, we also found that natural ACTH is different from corticosteroids on the level of gene transcription. Natural ACTH is an attractive therapeutic option for patients who may be problematic with NSAIDs, steroids or colchicine. Moreover, ACTH is nowadays widely available and inexpensive at least in China and most European countries(13). In our study, natural ACTH showed a lot difference from dexamethasone in terms of the transcription of inflammatory and metabolic related genes. It is still worth studying wether there is difference between natural ACTH and corticosteroids and whether natural ACTH can be used as a safe alternative to corticosteroids.

\section{Conclusion}

Through the collection of patient data and in vitro and in vivo experiments, we found that natural ACTH could alleviate the inflammatory response by inhibiting the aggregation of inflammatory cells and changing the function of macrophages, and verified that this effect is different from that of dexamethasone at the transcriptional level.

\section{Abbreviations}

ACTH: Adrenocorticotropic hormone

ANGPTL4: Angiopoietin-related protein 4

Arg-1: Arginase-1

ATP: adenosine-triphosphate

AXL: Anexelekto

BCL6: B-cell lymphoma 6 
CRF: corticotropin-releasing factors

DCFH-DA: 2, 7-Dichlorodi -hydrofluorescein diacetate

DDIT4: DNA Damage Inducible Transcript 4

ELISA: enzyme linked immunosorbent assay

FcyRIIIA: Low affinity immunoglobulin gamma Fc region receptor III-A

FITC: Fluorescein Isothiocyanate

GAS6: Growth arrest-specific protein 6

HE: hematoxylin-eosin staining

IL-1 $\beta$ : interleukin-1 $\beta$

IL1RN: Interleukin 1 Receptor Antagonist

IL-6: interleukin-6

IL-10: interleukin-10

IL-37: interleukin-37

iNOS: Nitric oxide synthase, inducible

MC-2R: melanocortin 2 receptor

MC-3R: melanocortin 3 receptor

MERTK: MER Proto-Oncogene, Tyrosine Kinase

MPO: Myeloperoxidase

MPTP: mitochondrial permeability transition pore

mTOR: mammalian target of rapamycin

MSU: monosodium urate

NLRP3: NLR Family Pyrin Domain Containing 3

NOX1: NADPH oxidase 1

NOX3: NADPH oxidase 1 
NR1H2: Nuclear Receptor Subfamily 1 Group H Member 2

NR3C1: Nuclear Receptor Subfamily 3 Group C Member 1, Glucocorticoid receptor

NSAIDs: Nonsteroidal Antiinflammatory Drugs

PBS: phosphate buffered saline

PDHA1: Pyruvate Dehydrogenase E1 Subunit Alpha 1

PDP1: Pyruvate Dehydrogenase Phosphatase 1

PGC-1a: Peroxisome proliferator-activated receptor gamma coactivator 1-alpha

PMA: Phorbol-12-myristate-13-acetate

PTX3: Pentraxin-related protein PTX3

qPCR: quantitative Real-time PCR

ROS: reactive oxygen species

RPMI: Roswell Park Memorial Institute

SMAD3: Mothers against decapentaplegic homolog 3

TGF- $\beta$ : transforming growth factor- $\beta$

THP1: Tohoku Hospital Pediatrics-1

TNF-a: Tumor Necrosis Factor- $a$

TYRO3: Tyrosine-protein kinase 3

VAS: visual analogue scale

VDR: Vitamin D3 receptor

XDH: Xanthine dehydrogenase/oxidase

\section{Declarations}

\section{Ethics approval and consent to participate}

Additional ethical approval was not required. 


\section{Consent for publication}

Not applicable.

\section{Availability of supporting data}

All data generated or analysed during this study are included in this published article and are publicly available.

Competing Interest

The authors declare no relevant conflicts of interest.

Funding

This work was supported by the National Natural Science Foundation of China (82071756 and 82071830).

Authors' informations

Division of Rheumatology, Huashan Hospital, Fudan University, Shanghai, China and Human Phenome Institute, Fudan University, 825 Zhangheng Road, Shanghai, China

Rui Xu, E-mail: 840420892@qq.com

Division of Rheumatology, Huashan Hospital, Fudan University, Shanghai, China

Li Zhao, E-mail: Izhao@unirheuma.org

Division of Rheumatology, Huashan Hospital, Fudan University, Shanghai, China Jiyu Liu, E-mail: jiyuliu@unirheuma.org

Division of Rheumatology, Huashan Hospital, Fudan University, Shanghai, China

Lin Cao, E-mail: 18702170230@163.com

Division of Rheumatology, Huashan Hospital, Fudan University, Shanghai, China, Institute of Rheumatology, Immunology and Allergy, Fudan University, Shanghai, China

Tianyi Zhao, E-mail: tyzhao@unirheuma.org

Division of Rheumatology, Huashan Hospital, Fudan University, Shanghai, China, Institute of Rheumatology, Immunology and Allergy, Fudan University, Shanghai, China

Yiyun Yu, E-mail: 13788978206@163.com 
Division of Rheumatology, Huashan Hospital, Fudan University, Shanghai, China, Institute of Rheumatology, Immunology and Allergy, Fudan University, Shanghai, China

Dandan Xuan, E-mail: xuandandan@fudan.edu.cn

Division of Rheumatology, Huashan Hospital, Fudan University, Shanghai, China, Institute of Rheumatology, Immunology and Allergy, Fudan University, Shanghai, China

Weiguo Wan, E-mail: 18121186085@163.com

Division of Rheumatology, Huashan Hospital, Fudan University, Shanghai, China, Institute of Rheumatology, Immunology and Allergy, Fudan University, Shanghai, China

Hejian Zou, E-mail: hjzou@unirheuma.org

Division of Rheumatology, Huashan Hospital, Fudan University, Shanghai, China, Institute of Rheumatology, Immunology and Allergy, Fudan University, Shanghai, China

Yu Xue, E-mail: yxue@unirheuma.org

Rui Xu and Li Zhao contributed to the work equally and should be regarded as co-first authors. Correspondence to Hejian Zou and Yu Xue.

Authors' contributions

All the authors are accountable for all aspects of the work in ensuring that questions related to the accuracy or integrity of any part of the work are appropriately investigated and resolved.

\section{Acknowledgments}

The co-first authors would like to acknowledge the suggestions provided by corresponding authors Hejian Zou and Yu Xue during the work.

\section{References}

1. Agudelo CA, Wise CM. Gout: diagnosis, pathogenesis, and clinical manifestations. Curr Opin Rheumatol. 2001;13:234-9.

2. Borghi C, Agabiti-Rosei E, et al. Hyperuricaemia and gout in cardiovascular, metabolic and kidney disease. Eur J Intern Med. 2020®80:1-11.

3. John D FitzGerald, Nicola Dalbeth, Ted Mikuls, Romina Brignardello-Petersen, Gordon Guyatt, Aryeh M Abeles, et al. 2020 American College of Rheumatology Guideline for the Management of Gout. Arthritis Care Res (Hoboken). 2020 ;72(6):744-760. 
4. M J Brownstein. Adrenocorticotropic hormone (ACTH) in the central nervous system. Adv Biochem Psychopharmacol. 1980;22:93-9.

5. Theresa Gillis et al. Repository corticotropin injection as adjunctive therapy in patients with rheumatoid arthritis who have failed previous therapies with at least three different modes of action. Open Access Rheumatology: Research and Review. 2017, 9:131-138.

6. Peter A Fischer et al. Repository corticotropin injection in patients with rheumatoid arthritis resistant to biologic therapies. Open Access Rheumatology: Research and Reviews. 2018:10 13-19.

7. JJ Fiechtner et al. Treatment of moderately to severely active systemic lupus erythematosus with adrenocorticotropic hormone: a single-site, open-label trial. Lupus. 2014; 23: 905-912.

8. Furie Ret al. Efficacy and tolerability of repository corticotropin injection in patients with persistently active SLE: results of a phase 4, randomised, controlled pilot study. Lupus Science \& Medicine. 2016;3:e000180.

9. Gutman AB, Yu TF. Effects of adrenocorticotropic hormone (ACTH) in gout. Am J Med. 1950;9:2430.

10. Axelrod D, Preston S. Comparison of parenteral adrenocorticotropic hormone with oral indomethacin in the treatment of acute gout. Arthritis Rheum. 1988;31:803-5.

11. Ritter J, Kerr LD, Valeriano-Marcet J, Spiera H. ACTH revisited: effective treatment for acute crystal induced synovitis in patients with multiple medical problems. J Rheumatol. 1994;21:696-9.

12. Siegel LB, Alloway JA, Nashel DJ. Comparison of adrenocorticotropic hormoneand triamcinolone acetonide in the treatment of acute gouty arthritis. J Rheumatol. 1994;21:1325-7.

13. Dimitrios Daoussis et al. ACTH as first line treatment for acute gout in 181 hospitalized patients. Joint Bone Spine.2013;80(3) : 291-4.

14. Dimitrios Daoussis et al. ACTH as a treatment for acute crystal-induced arthritis: update on clinical evidence and mechanisms of action. Seminars in Arthritis and Rheumatism. 2014;43(5):648-53.

15. Munck, A., P. M. Guyre, and N. J. Holbrook. 1984. Physiological functions of glucocorticoids in stress and their relation to pharmacological actions. Endocr. 1984;5(1):25-44.

16. Lei Liu, Xiaoxia Zhu et al. Sirt1 ameliorates monosodium urate crystal-induced inflammation by altering macrophage polarization via the PI3K/Akt/STAT6 pathway. Rheumatology. 2019;58(9):16741683.

17. Samanta C Funes, Mariana Rios, Jorge Escobar-Vera, Alexis M Kalergis. Implications of macrophage polarization in autoimmunity. Immunology. 2018 Jun;154(2):186-195.

18. Jaroslava Chylikova, Jana Dvorackova et al. M1/M2 macrophage polarization in human obese adipose tissue. Biomed Pap Med Fac Univ Palacky Olomouc Czech Repub. 2018;162(2):79-82.

19. Van den Bossche, J., O’Neill, L.A., Menon, D. Macrophage Immunometabolism: Where Are We (Going)? Trends Immunol. 2017;38:395-406.

20. Rambold, A.S., Pearce, E.L. Mitochondrial Dynamics at the Interface of Immune Cell Metabolism and Function. Trends Immunol. 2018;39: 6-18. 
21. Ryan, D.G., Murphy, M.P., Frezza, C., Prag, H.A., Chouchani, E.T., O’Neill, L.A., Mills, E.L. Coupling Krebs cycle metabolites to signalling in immunity and cancer. Nat. Metab. 2019;1: 16-33.

22. O'Neill, L.A., Kishton, R.J., and Rathmell, J.A guide to immunometab- olism for immunologists. Nat. Rev. Immunol. 2016;16: 553-565.

23. Andrew $\mathrm{P}$ Halestrap. What is the mitochondrial permeability transition pore? J Mol Cell Cardiol. 2009;46(6):821-31.

24. Gisela Beutner, Kambiz N Alavian et al. The Mitochondrial Permeability Transition Pore and ATP Synthase. Handb Exp Pharmacol. 2017;240:21-46.

25. Hsin-Ying Wu, Chung-Hsiung Huang et al. Cannabidiol induced apoptosis in human monocytes through mitochondrial permeability transition pore-mediated ROS production. Free Radic Biol Med. $2018 ; 124: 311-318$.

26. Jessica Maiuolo, Francesca Oppedisano et al. Regulation of uric acid metabolism and excretion. Int J Cardiol. 2016 15;213:8-14.

27. Merih Oray, Khawla Abu Samra et al. Long-term side effects of glucocorticoids. Expert Opin Drug Saf. 2016;15(4):457-65.

28. Binod Aryal, Nathan L Price, Yajaira Suarez, Carlos Fernández-Hernando. ANGPTL4 in Metabolic and Cardiovascular Disease. Trends Mol Med. 2019 ;25(8):723-734.

29. Jun Fan, Changliang Shan, Hee-Bum Kang, Shannon Elf, Jianxin Xie, Meghan Tucke. Tyr phosphorylation of PDP1 toggles recruitment between ACAT1 and SIRT3 to regulate the pyruvate dehydrogenase complex. Mol Cell. 2014 Feb 20;53(4):534-48.

30. Nicolas Venteclef, Tomas Jakobsson, Anna Ehrlund, Anastasios Damdimopoulos, Laura Mikkonen, Ewa Ellis, et al. GPS2-dependent corepressor/SUMO pathways govern anti-inflammatory actions of LRH-1 and LXRbeta in the hepatic acute phase response. Genes Dev. 2010;24(4):381-95.

31. Giulio Cavalli, Charles A Dinarello. Suppression of inflammation and acquired immunity by IL-37. Immunol Rev. $2018 ; 281(1): 179-190$.

32. Bishuang Cai, Paola Dongiovanni et al. Macrophage MerTK Promotes Liver Fibrosis in Nonalcoholic Steatohepatitis. Cell Metabolism. 2020;31(2):406-421.

33. Shuang Qi, Feng Zhao, Zinan Li, Feng Liang, Shanshan Yu. Silencing of PTX3 alleviates LPS-induced inflammatory pain by regulating TLR4/NF-KB signaling pathway in mice. Biosci Rep. 2020 Feb 28;40(2):BSR20194208.

34. Mizushima T., Yagi H., Takemoto E., Shibata-Koyama M., Isoda Y., lida S., et al. Structural basis for improved efficacy of therapeutic antibodies on defucosylation of their Fc glycans. Genes Cells. 2011;16:1071-1080.

35. Rebecca J Leeman-Neill, Govind Bhagat. BCL6 as a therapeutic target for lymphoma. Expert Opin Ther Targets. 2018;22(2):143-152.

36. Zhi-Feng Miao, Jing-Xu Sun, Mahliyah Adkins-Threats, Min-Jiao Pang, Jun-Hua Zhao, Xin Wang, et al. DDIT4 Licenses Only Healthy Cells to Proliferate During Injury-induced Metaplasia. 
Gastroenterology.2021;160(1):260-271.

37. Knutti D., Kaul A., Kralli A. A tissue-specific coactivator of steroid receptors, identified in a functional genetic screen. Mol. Cell. Biol. 2000;20:2411-2422.

38. Wang G., Matsuura I., He D., Liu F. Transforming growth factor-\{beta\}-inducible phosphorylation of Smad3. Biol. Chem. 2009;284:9663-9673.

39. Park I.K., Giovenzana C., Hughes T.L., Yu J., Trotta R., Caligiuri M.A. The Axl/Gas6 pathway is required for optimal cytokine signaling during human natural killer cell development. Blood. 2009;113:2470-2477.

\section{Figures}


Fig.1

A

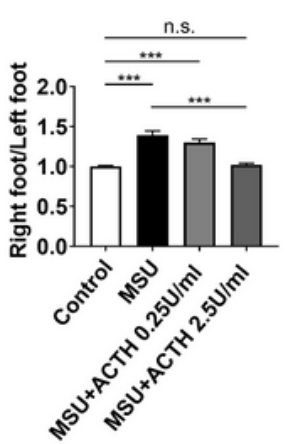

C

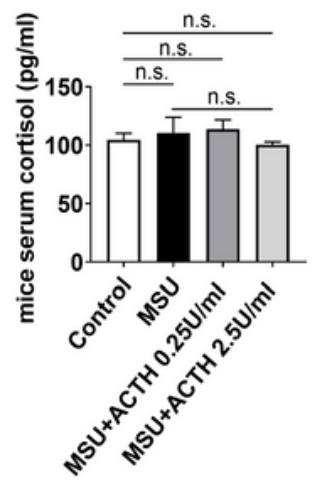

B
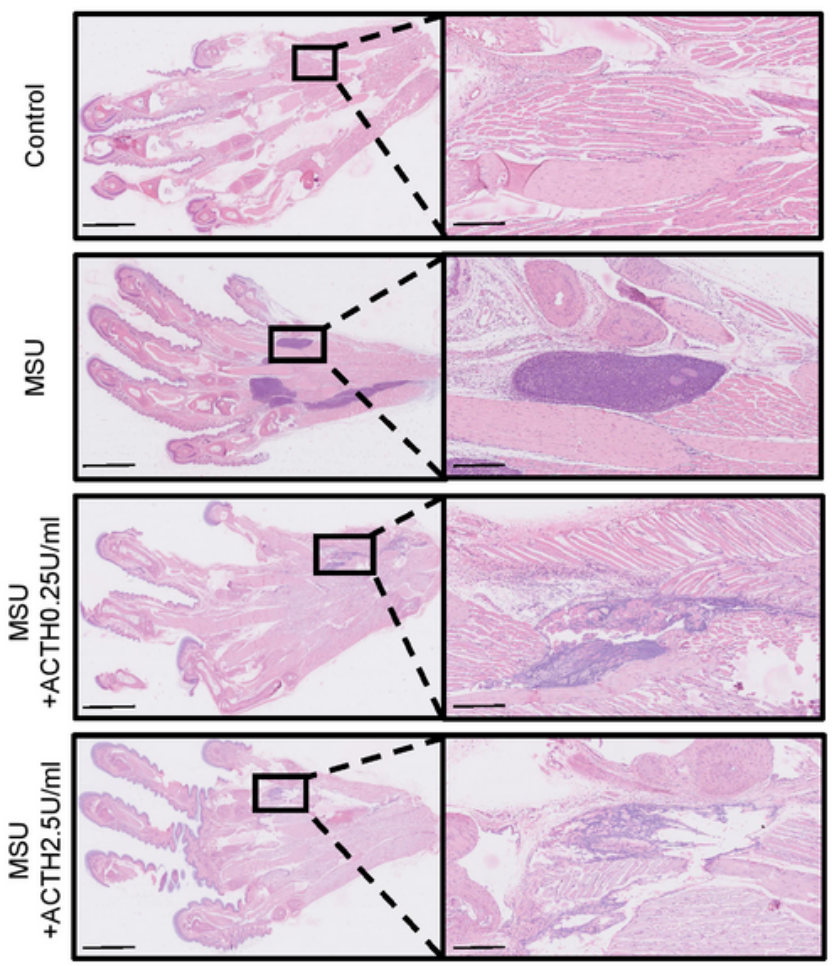

\section{Figure 1}

Natural ACTH can alleviate the swelling and inflammation of mice paw. Except for the control group, mice in other groups were injected with $1 \mathrm{mg} \mathrm{MSU}$ crystal suspension from the joints of right hind paw and with equal saline into the other side. Natural ACTH was injected with $800 \mu l$ per mouse subcutaneously 30 minutes after. Blood was collected 8 hours later and the mice were executed. Collected blood was entrifuged at a speed of $3000 \mathrm{r}, 15$ minutes to collect the serum, with the temperature being kept at $4{ }^{\circ} \mathrm{C}$ 
during the whole process. A: Before treatment, the joint thickness of hind paw was measured for 3 times by the caliper. Average value was calculated and recorded. The thickness was measured once again after 8 hours and was compared with the value measured before treatment. The swelling of paw was signified by the ratio of average right paw thickness to that of left paw. B: Histological changes of the two-side

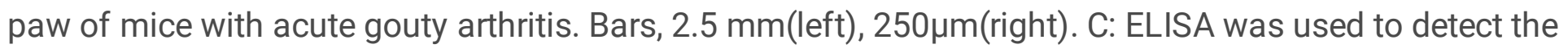
level of cortisol in mice serum $(\mathrm{pg} / \mathrm{ml}) . \mathrm{n}=3$. Data are mean \pm SEM and are representative of two independent experiments. ${ }^{*}, \mathrm{P}<0.05 ; * \star, \mathrm{P}<0.01{ }^{\star \star * \star}, \mathrm{P}<0.001$ ฟtwo-tailed unpaired Student's $\mathrm{t}$ test $(\mathrm{A}$ and C) $₫$ n.s., no significance. 
Fig.2

A
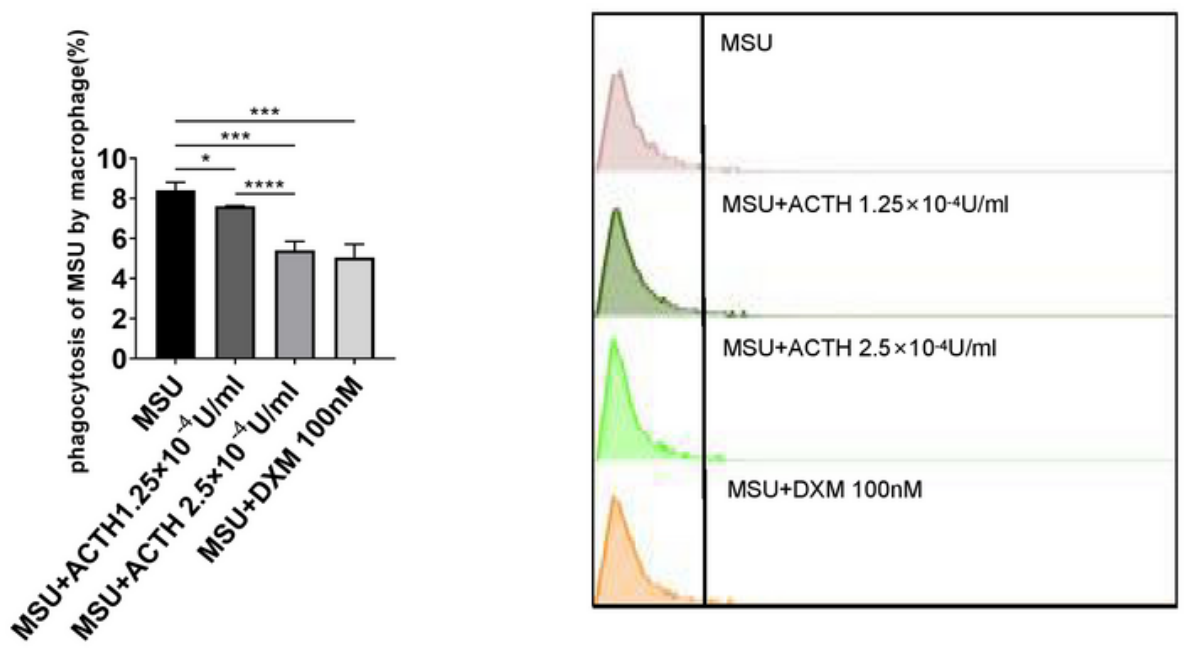

B
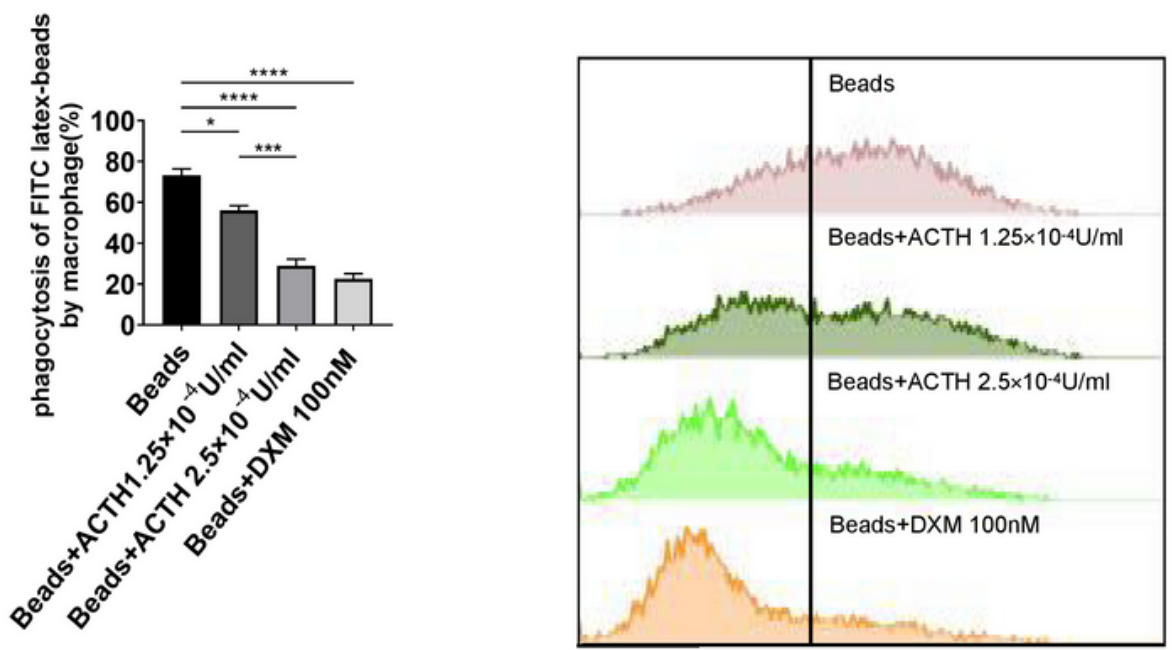

\section{Figure 2}

Natural ACTH can inhibit the phagocytosis of THP1 cells. A: $100 \mu \mathrm{g} / \mathrm{ml}$ MSU crystal was added into THP1 cells for stimulation. $1.25 \times 10-4 \mathrm{U} / \mathrm{ml}, 2.5 \times 10-4 \mathrm{U} / \mathrm{ml}$ natural ACTH and $100 \mathrm{nM}$ dexamethasone were applied 30 minutes later, respectively. Flow cytometry was used for detection after 48 hours. B: THP1 cells were incubated with 2.5\% FITC-latexbeads for 30 minutes, and then added with natural ACTH and dexamethasone at the same concentration described in A. Flow cytometry was used for detection 
after 48 hours. Data are mean \pm SEM and are representative of at least three independent experiments. *, $P<0.05 ; * \star, P<0.01 ; * \star \star, \quad P<0.001$, ${ }^{\star \star \star \star}, P<0.0001$; two-tailed unpaired Student's t test $\$ n.s., no significance.

Fig.3

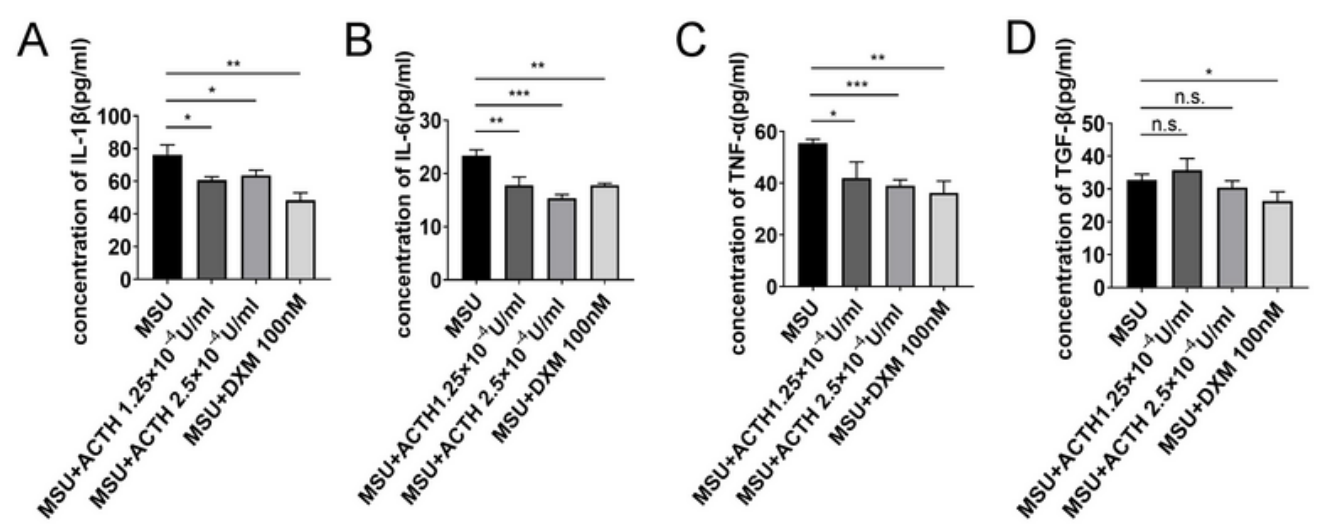

E

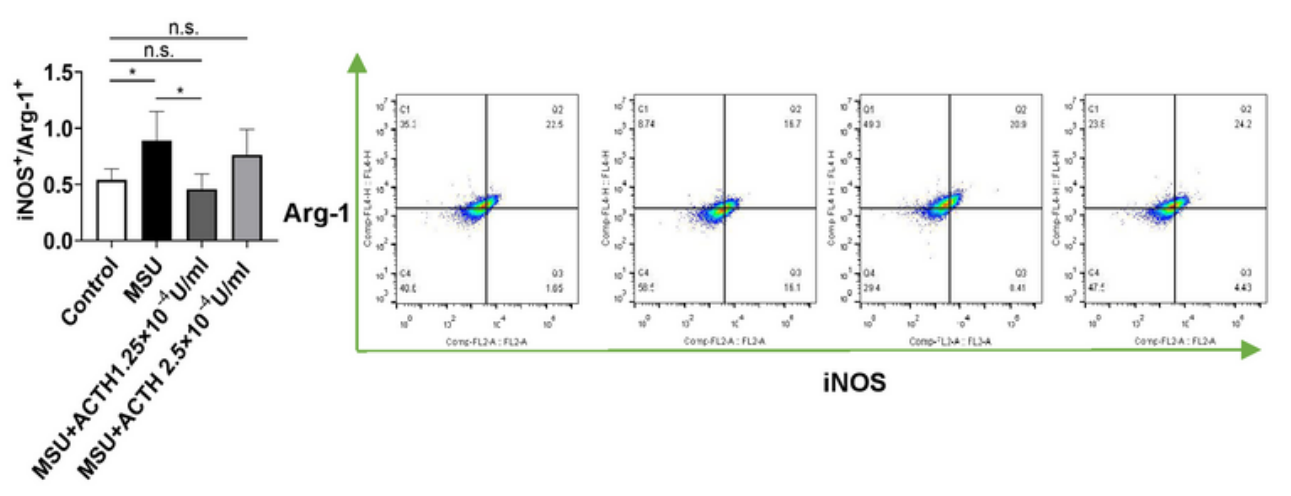

Figure 3

M1 type Macrophage can be turned into anti-inflammatory trend of M2 type by natural ACTH. A: 100 $\mu \mathrm{g} / \mathrm{ml}$ MSU crystal was used to stimulate THP1 cells. $1.25 \times 10-4 \mathrm{U} / \mathrm{ml}, 2.5 \times 10-4 \mathrm{U} / \mathrm{ml}$ natural ACTH and 
$100 \mathrm{nM}$ dexamethasone were added repsectively 30 minutes later. Cell supernatant was collected after 48 hours. ELISA was used to detect the concentration of IL-1 $1 \beta$, IL-6, TNF- $\alpha$ and TGF- $\beta$. B: $100 \mu \mathrm{g} / \mathrm{ml} \mathrm{MSU}$ crystal was used to stimulate THP1 cells. $1.25 \times 10-4 \mathrm{U} / \mathrm{ml}, 2.5 \times 10-4 \mathrm{U} / \mathrm{ml}$ natural ACTH and $100 \mathrm{nM}$ dexamethasone were added repsectively 30 minutes later. Cell supernatant was collected after 48 hours, and flow cytometry was used for detection. The trend of polarization was determined by the ratio of iNOS fluorescence intensity to that of Arg- 1 . Data are mean \pm SEM and are representative of three independent experiments. *, $\mathrm{P}<0.05$; $* \star, \mathrm{P}<0.01$; $* \star \star, \mathrm{P}<0.001$; two-tailed unpaired Student's $\mathrm{t}$ test; $\mathrm{n} . \mathrm{s}$., no significance. 
Fig.4

A

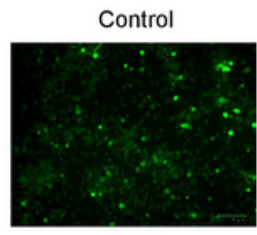

MSU

$+\mathrm{ACTH} 1.25 \times 10^{-4} \mathrm{U} / \mathrm{ml}$

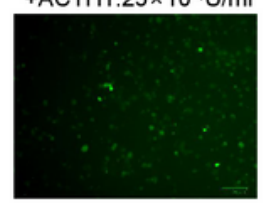

MSU+DXM 100nM
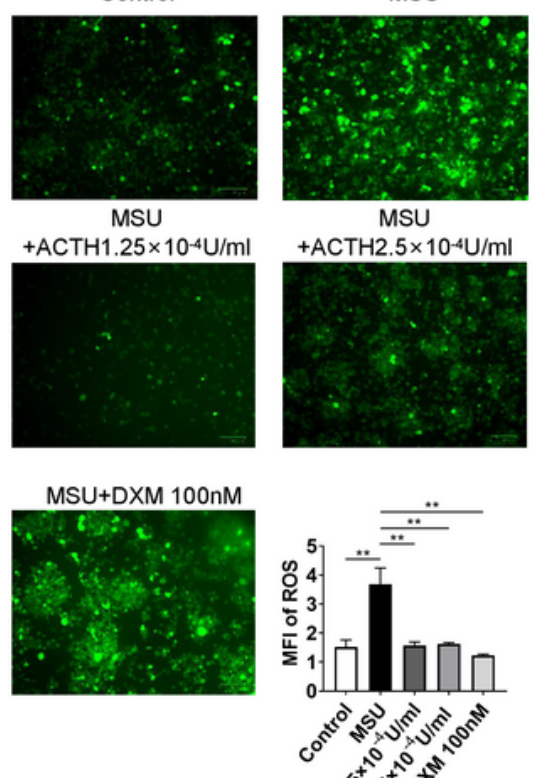

$+\mathrm{ACTH} 2.5 \times 10^{-4} \mathrm{U} / \mathrm{ml}$
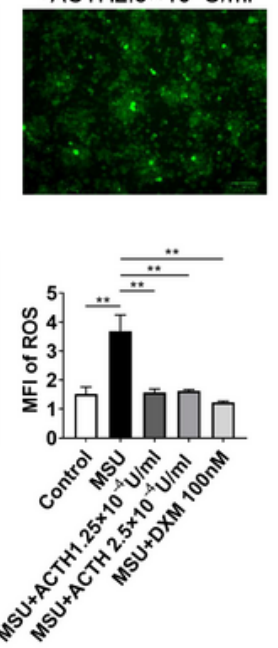

C

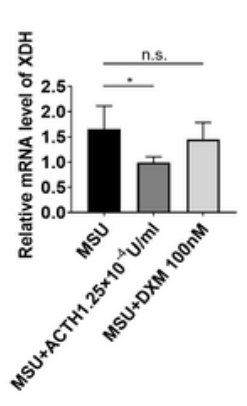

D

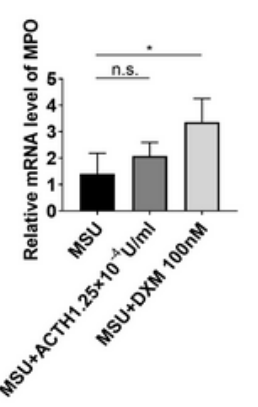

B

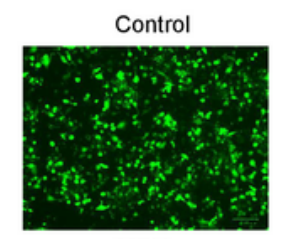

MSU
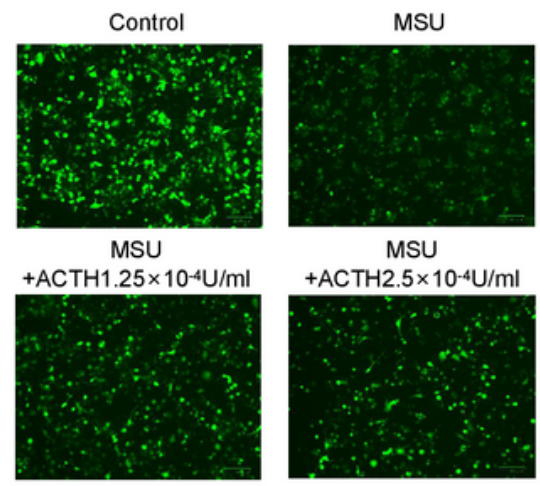

MSU

$+\mathrm{ACTH} 2.5 \times 10^{-4} \mathrm{U} / \mathrm{ml}$
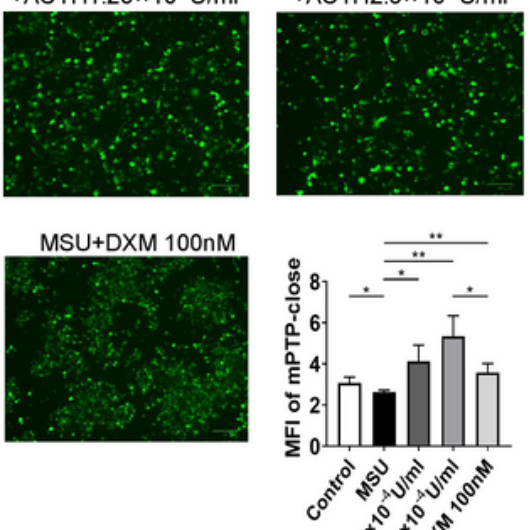

E

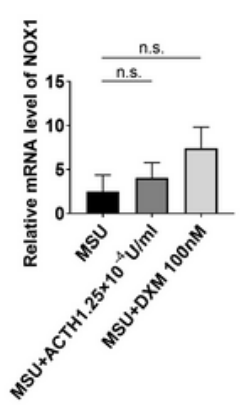

$\mathrm{F}$

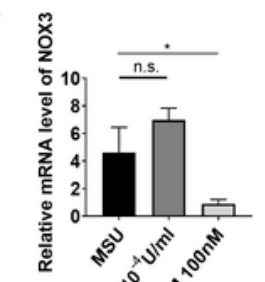

\section{Figure 4}

Natural ACTH downregulates the ROS level of macrophage and inhibits the opening of MPTP. A: 100 $\mu \mathrm{g} / \mathrm{ml} \mathrm{MSU}$ crystal was added to stimulate the cells for 30 minutes, and then $1.25 \times 10-4 \mathrm{U} / \mathrm{ml}, 2.5 \times 10-4$ $\mathrm{U} / \mathrm{ml}$ natural ACTH and $100 \mathrm{nM}$ dexamethasone were applied respectively for 48 hours. Finally, cells were collected for DCFH-DA staining, fluorescence microscopy and flow cytometry. B: qPCR was used to detect the expression of related genes. C: Calcein AM staining was conducted after stimulation, the condition of 
which is just like that mentioned in A. The results, analyzed by fluorescence microscope and flow cytometry, showed fluorenscence intensity was negatively correlated with the damaged mitochondrial function. Bars, $100 \mu \mathrm{m}$. Data are mean \pm SEM and are representative of three independent experiments. *, $\mathrm{P}<0.05$; **, $\mathrm{P}<0.01$; two-tailed unpaired Student's t test; n.s., no significance.

Fig.5

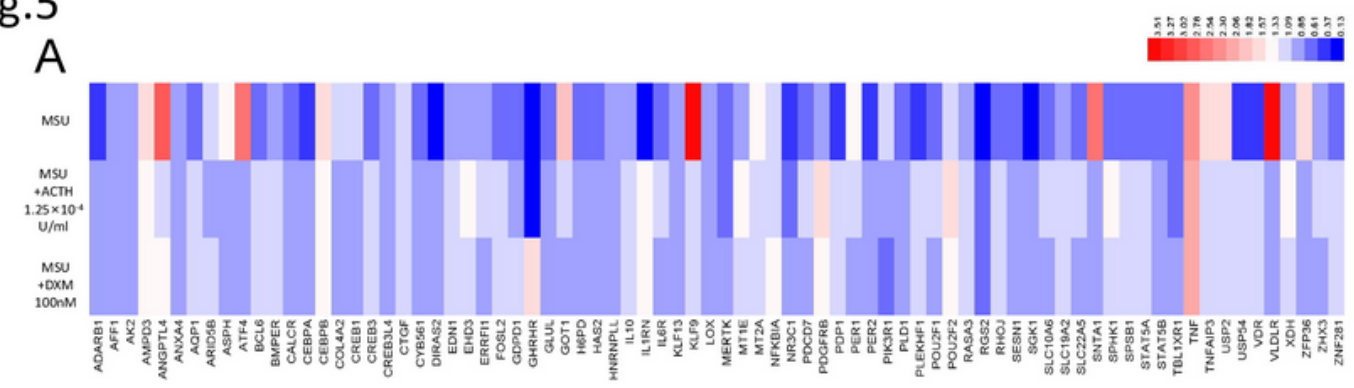

\section{B}

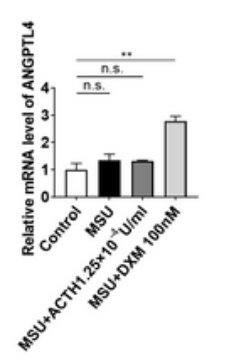

$\mathrm{C}$
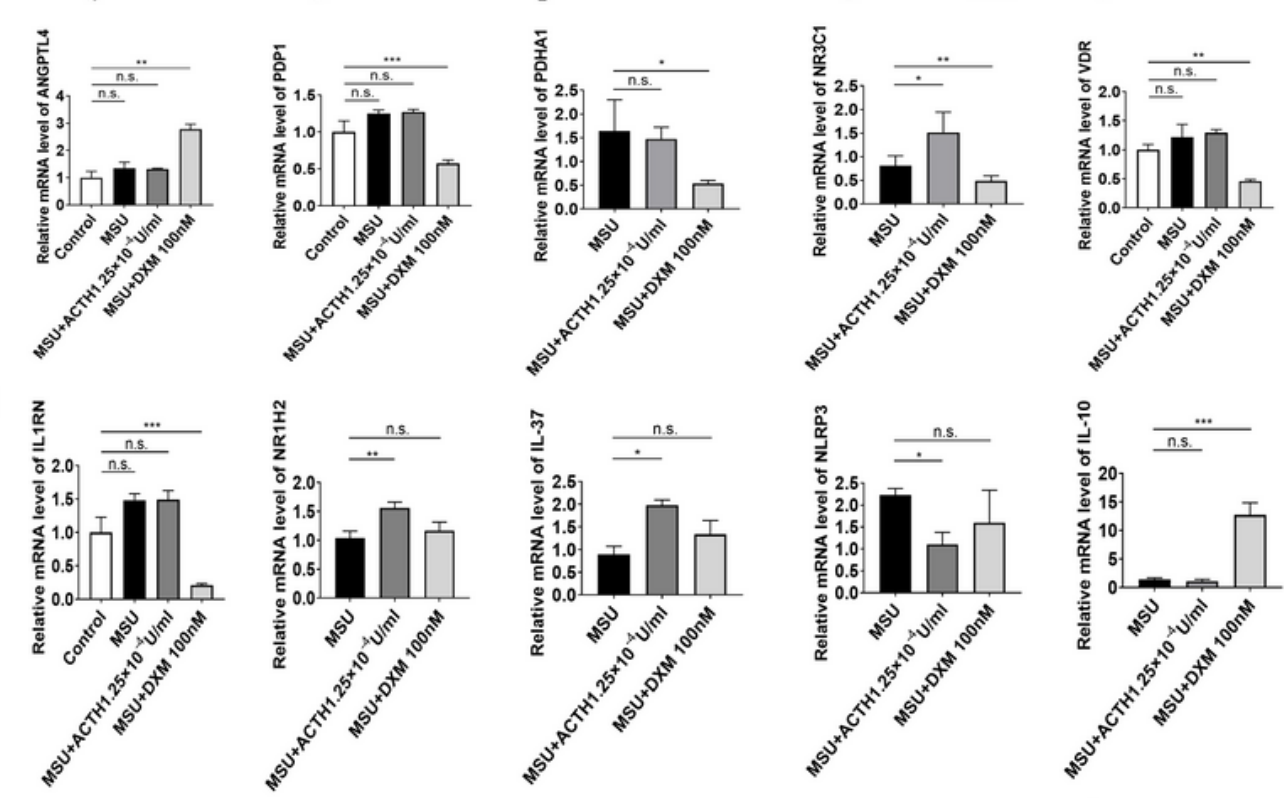

$\mathrm{D}$
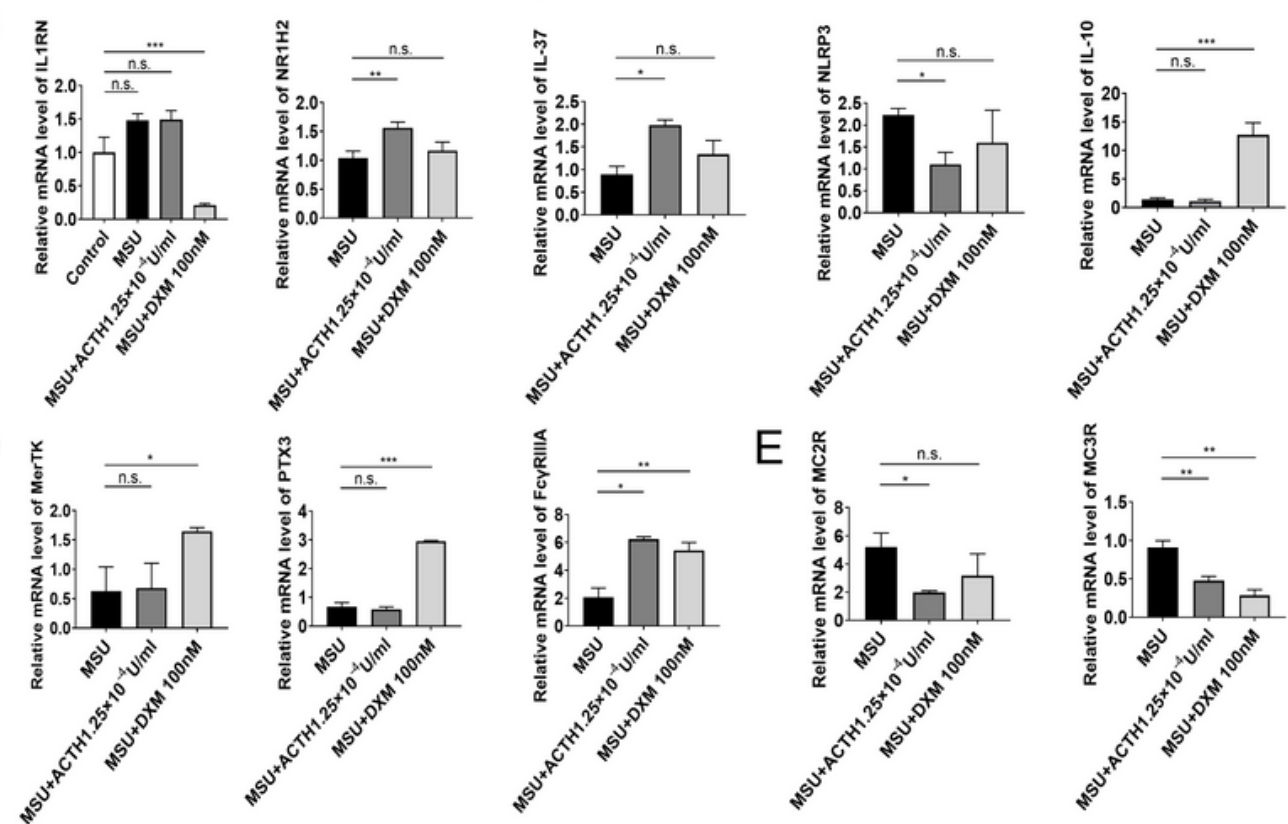

$\mathrm{F}$

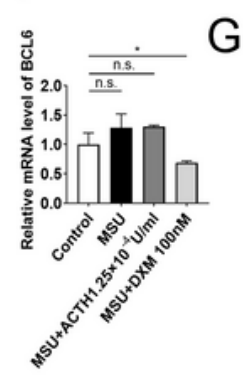

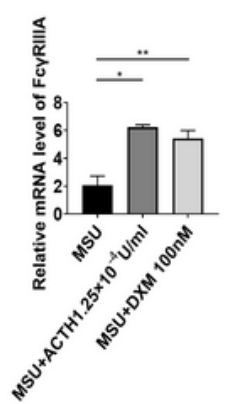

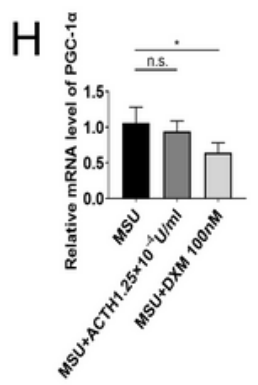

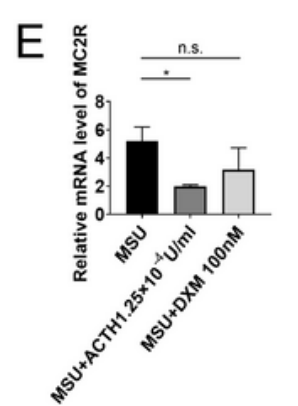
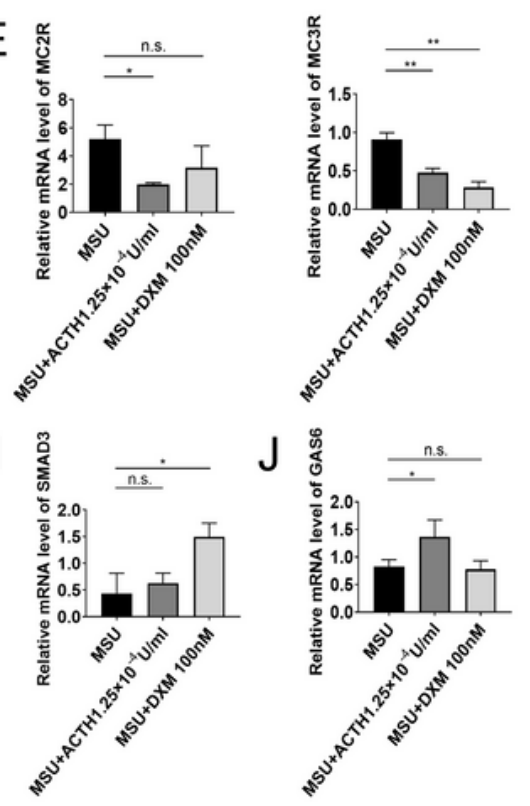

Figure 5 
Natural ACTH is different from dexamethasone in terms of the transcription of Inflammatory-related genes. A. Gene heat map showing the effect of natural ACTH and dexamethasone on the gene transcription of THP1 cells which were stimulated by MSU crystal. B-J: Expression of some related genes . Data are mean \pm SEM and are representative of three independent experiments. ${ }^{*}, P<0.05 ; * \star, P<0.01$;

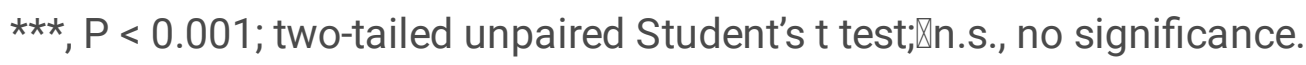

\section{Supplementary Files}

This is a list of supplementary files associated with this preprint. Click to download.

- Additionalfile1.pdf 